\title{
Simplified Calculus for the Design of a Cryogenic Current Comparator
}

\author{
Javier Sesé, Elena Bartolomé, Agustín Camón, Jaap Flokstra, Gert Rietveld, and Conrado Rillo
}

\begin{abstract}
The calculation of inductances of superconducting structures like the cryogenic current comparator (CCC) is not straightforward due to image effects and unknown current distributions. By treating the problem as a magnetic circuit, an approximate analytical expression for the self-inductance of the shielded CCC is obtained. This formula can be used to derive a "rule of thumb" for maximizing the value of an inductance inside a superconducting shield. Using this rule the design of an optimum CCC is simplified.
\end{abstract}

Index Terms-Cryogenic current comparator (CCC), inductance calculations, SQUID, superconductors.

\section{INTRODUCTION}

$\mathbf{T}$ HE cryogenic current comparator (CCC) [1] allows the control of the ratio of two currents $I_{1} / I_{2}=N_{2} / N_{1}$ (where $N_{1}$ and $N_{2}$ are integer numbers) with high precision. Resistance bridges based on the $\mathrm{CCC}$ allow resistance comparisons to be made at the highest metrological level. They are, at present, widely used to transfer the value of the quantum Hall resistance standard to very stable resistors [2]. More recently, the use of a CCC toward the realization of a quantum current standard is being investigated [3]-[5]. There, it will be used for the accurate amplification of quantized currents, in the pA range, produced by single electron tunneling devices.

\section{DeSIGN OF AN Optimum CCC}

The ultimate current resolution of a CCC per turn in the primary windings that is ideally coupled to a SQUID is given by [6]

$$
\left\langle i_{p}^{2}\right\rangle=\frac{8 \varepsilon_{S Q}}{k_{S Q}^{2} L_{\mathrm{CCC}, \mathrm{eff}}}
$$

where $\varepsilon_{S Q} / k_{S Q}^{2}$ is the coupled energy resolution of the SQUID and $L_{\mathrm{CCC} \text {,eff }}$ is the effective inductance of the CCC overlapped tube inside the superconducting shield (Fig. 1). The situation of maximum coupling (optimum sensitivity) occurs if the selfinductances of the sensing coil and the SQUID input coil $L_{i}$ match. This matching condition can be closely achieved by constructing a sensing coil with $N_{S}$ turns wound very close to the overlapped tube with $N_{S}=\sqrt{L_{i} / L_{\mathrm{CCC}, \mathrm{eff}}}$.

Manuscript received June 17, 2002; revised October 21, 2002.

J. Sesé, E. Bartolomé, and J. Flokstra are with the Low Temperature Division, Applied Physics Department, University of Twente, Enschede, The Netherlands (e-mail: j.sese@tn.utwente.nl).

A. Camón and C. Rillo are with the Institute of Material Science of Aragon (ICMA), CSIC-University of Zaragoza, Zaragoza, Spain.

G. Rietveld is with the Department of Electricity and Magnetism, NMi Van Swinden Laboratorium, Delft, The Netherlands.

Digital Object Identifier 10.1109/TIM.2003.811579

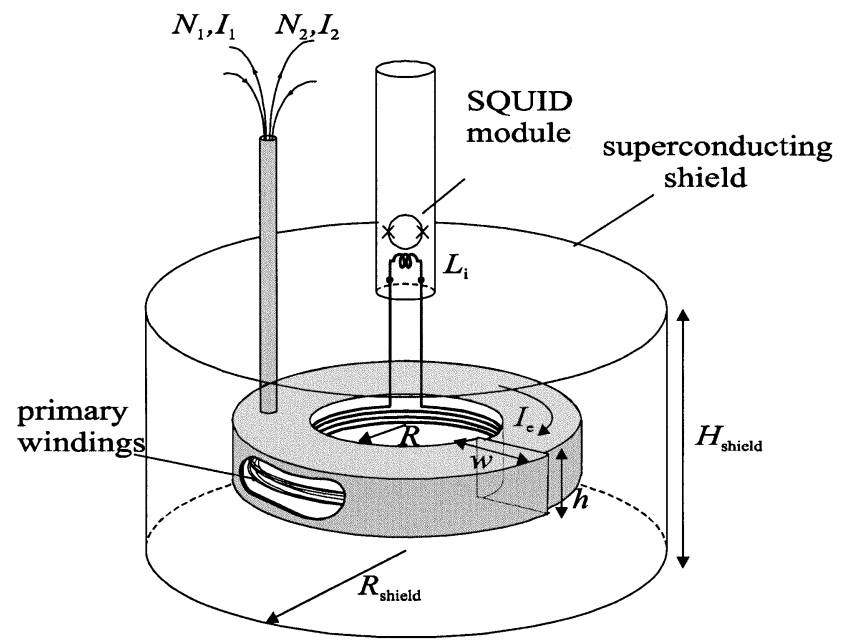

Fig. 1. Schematics of a CCC. The overlapped tube has cross section $h \cdot w$ and internal radius $R$. The flux created by the Meissner current $I_{e}=N_{1} I_{1}-N_{2} I_{2}$ is picked up by a sensing coil connected to a SQUID. The whole is surrounded by a superconducting shield with dimensions $R_{\text {shield }}$ and $H_{\text {shield }}$.

For a given number of turns, the cross section $h \cdot w$ is fixed and the optimum CCC will be the one with maximum $L_{\mathrm{CCC}, \text { eff }}$ allowed in the available diameter. The optimization of $L_{\mathrm{CCC}, \text { eff }}$ with respect to the shields, based on different numerical methods, has been the subject of several papers [7]-[10]. Numerical methods can be applied to any geometry and have proven to give accurate results but are time-consuming, not straightforward, and the underlying physics is hardly seen. It would be desirable to have an approximate analytical formula that is valid for the typical dimensions of a CCC. In this work, such a formula is derived using the theory of magnetic circuits.

\section{ShIElded CCC AS A MAGnetiC CiRCUIT}

Magnetic circuit theory (see, for example, [11]) establishes a perfect analogy between the conduction of magnetic flux through a medium with permeability $\mu$ and the conduction of current through a medium of conductivity $\sigma$, where the two media have the same geometry. Fig. 2 shows this analogy. On the left side of Fig. 2, a current circulating in the overlapping tube creates a magnetic field that is contained inside the superconducting shield. The right part of Fig. 2 represents a solid piece of metal with the same dimensions as the space between the superconducting shield and the overlapping tube. A small slit has been introduced that allows a voltage difference $V$ to be applied. The distribution of the current density inside the piece of metal is the same as the distribution of magnetic field in the previous case. The analogous quantity to the electrical 


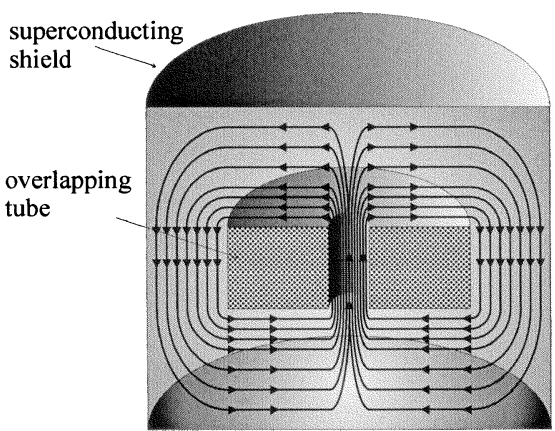

(a)

Fig. 2. Equivalence between (a) magnetic and (b) electrical circuits.

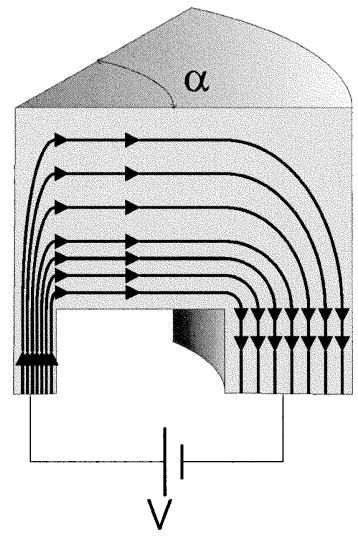

Fig. 3. Section of Fig. 2(b) for the calculation of electrical resistance. This section has electrical resistance that is $\pi / \alpha$ [radians] times the resistance of Fig. 2(b).

resistance $R_{e l}$ is the magnetic reluctance $\Re$. Even more, a magnetic circuit can be considered as a parallel or series connection of reluctances. For our case, the self-inductance $L$ of the magnetic circuit is the inverse of its reluctance. $L, \Re$, and $R_{e l}$ are related by

$$
L=\frac{1}{\Re}=\frac{\mu_{0}}{\sigma R_{e l}}
$$

where $\mu_{0}$ is the permeability of vacuum and $\sigma$ is the conductivity of the metal used. Hence, with the magnetic circuit theory, it is possible to deduce the self-inductance of a superconducting structure by making an electrical resistance measurement. Note that taking advantage of the symmetry, a simple piece like the one in Fig. 3 would be sufficient for performing the calculations. Nevertheless, for the design of a CCC it is still desirable to have an approximate analytical expression for the calculation of $L_{\text {CCC,eff }}$.

\section{ANALYTICAL EXPRESSION FOR THE EFFECTIVE SELF-INDUCTANCE}

In order to find an analytical expression, the magnetic circuit has been split in several pieces that approximate the real geometry. These are shown in Fig. 4.

Hence, our formula for $L_{\mathrm{CCC} \text {,eff }}$ is

$$
L_{\mathrm{CCC}, \mathrm{eff}}=\frac{1}{\Re_{1}+\Re_{2}+2 \Re_{3}} .
$$

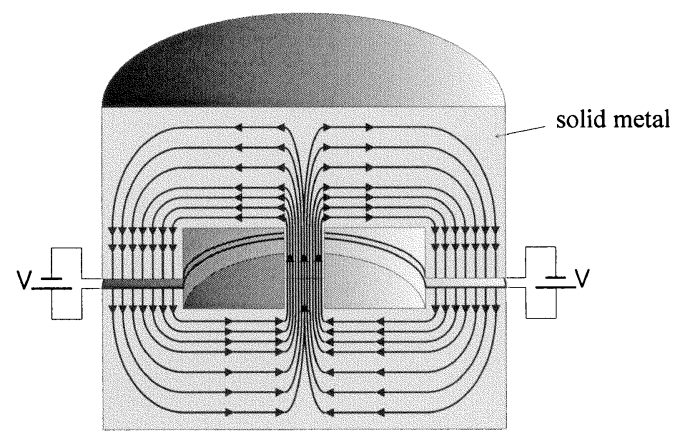

(b)
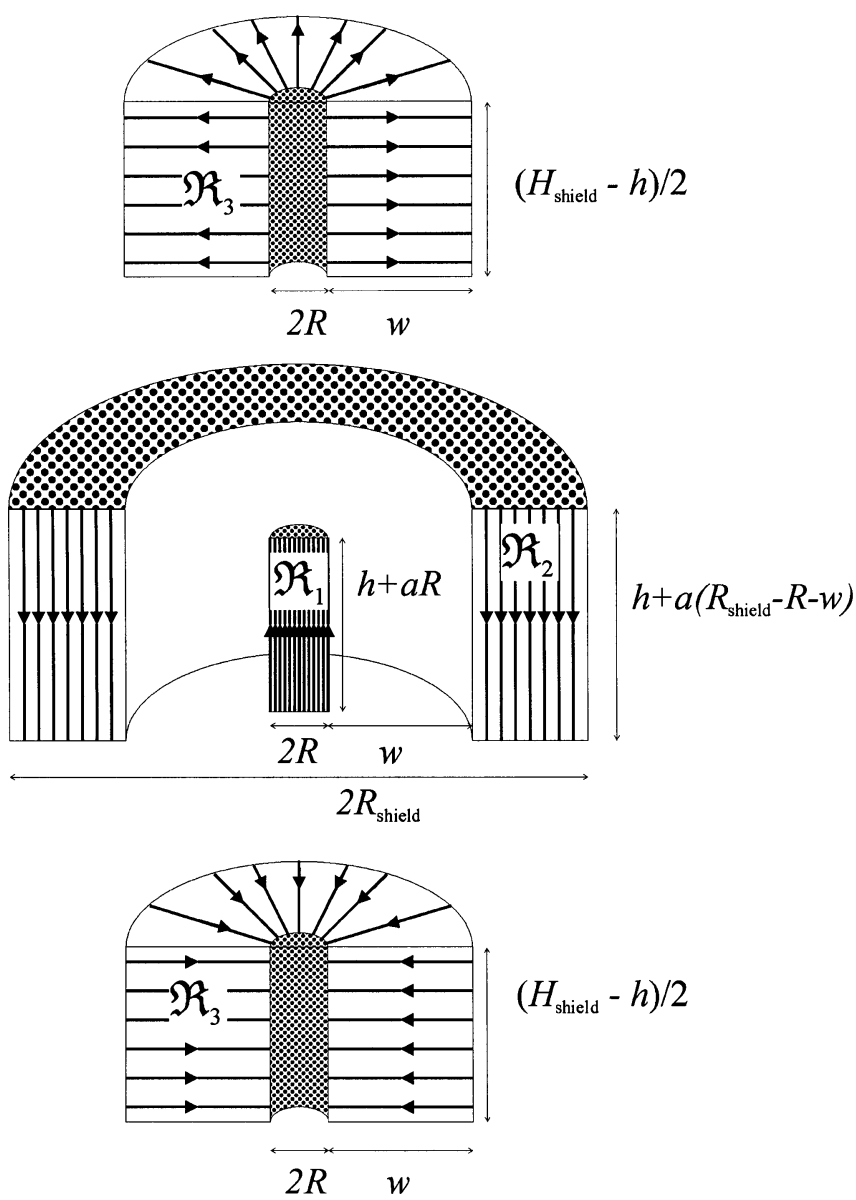

Fig. 4. Section of the schematics of Fig. 2 for the calculation of the reluctance. $\Re_{1}$ and $\Re_{2}$ are approximations for the reluctance of the central hole and the space between the overlapping tube and the superconducting shield, respectively. $\Re_{3}$ is the approximation of the reluctance for the top and bottom parts of Fig. 2. The arrows indicate the direction of flow of the magnetic flux.

When the magnetic field is homogeneous, the reluctance of a piece can be calculated as $\Re=l / \mu_{0} S$ where $l$ and $S$ are the length and the cross section of the piece. A homogeneous magnetic field is expected to be a good approximation when the dimensions $h$ and $w$ are of the same order of magnitude as $R_{\text {shield }}$ and $H_{\text {shield. }}$. Hence, with this assumption, $\Re_{1}$ and $\Re_{2}$ are expressed as

$$
\Re_{1}=\frac{h+a R}{\mu_{0} \pi R^{2}} ; \quad \Re_{2}=\frac{h+a\left(R_{\text {shield }}-R-W\right)}{\mu_{0} \pi\left(R_{\text {shield }}^{2}-(R+W)^{2}\right)} .
$$


TABLE I

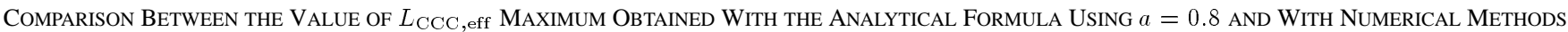

\begin{tabular}{|c|c|c|c|c|c|c|c|}
\hline \multirow[b]{2}{*}{$H_{\text {shield }}(\mathrm{mm})$} & \multirow[b]{2}{*}{$R_{\text {shield }}(\mathrm{mm})$} & \multirow[b]{2}{*}{$h(\mathrm{~mm})$} & \multirow[b]{2}{*}{$w(\mathrm{~mm})$} & \multirow[b]{2}{*}{$R_{\text {opt }}(\mathrm{mm})$} & \multicolumn{2}{|c|}{$L_{\mathrm{CCC}, \text { eff }}$ maximum $(\mathrm{nH})$} & \multirow[b]{2}{*}{ difference (\%) } \\
\hline & & & & & analytical & numerical & \\
\hline 80 & 30 & 10 & 10 & 15 & 24.18 & 22.65 & 6.3 \\
\hline 80 & 40 & 10 & 10 & 23 & 41.76 & 40.60 & 2.8 \\
\hline 80 & 50 & 10 & 10 & 31 & 60.55 & 60.90 & -0.6 \\
\hline 80 & 60 & 10 & 10 & 39 & 80.10 & 82.90 & -3.5 \\
\hline 80 & 30 & 15 & 15 & 12 & 13.27 & 12.30 & 7.3 \\
\hline 80 & 40 & 15 & 15 & 19 & 27.01 & 25.80 & 4.5 \\
\hline 80 & 50 & 15 & 15 & 27 & 42.51 & 41.80 & 1.7 \\
\hline 80 & 60 & 15 & 15 & 35 & 58.94 & 59.30 & -0.6 \\
\hline 80 & 30 & 20 & 20 & 8 & 6.16 & 5.80 & 5.9 \\
\hline 80 & 40 & 20 & 20 & 16 & 17.06 & 16.20 & 5.0 \\
\hline 80 & 50 & 20 & 20 & 23 & 29.73 & 29.10 & 2.1 \\
\hline 80 & 60 & 20 & 20 & 31 & 43.65 & 43.60 & 0.1 \\
\hline 100 & 30 & 20 & 20 & 8 & 6.27 & 5.85 & 6.7 \\
\hline 100 & 40 & 20 & 20 & 16 & 17.57 & 16.25 & 7.5 \\
\hline 100 & 50 & 20 & 20 & 23 & 30.95 & 29.31 & 5.3 \\
\hline 100 & 60 & 20 & 20 & 31 & 45.75 & 44.31 & 3.2 \\
\hline 30 & 40 & 20 & 20 & 16 & 10.77 & 11.30 & -4.9 \\
\hline 40 & 40 & 20 & 20 & 16 & 13.83 & 14.60 & -5.6 \\
\hline 200 & 40 & 20 & 20 & 16 & 18.50 & 17.65 & 4.6 \\
\hline
\end{tabular}

The parameter $a$ is of the order of 1 and it takes into account the addition of some reluctance in the corners where the flux lines have to bend. An approximate value of $a$ will be given in Section V. $\Re_{3}$ is a piece with a variable cross section, hence the reluctance is the result of an integral $\Re=\int\left(1 / \mu_{0} S(l)\right) d l$ and it is given by

$$
\Re_{3}=\frac{\ln \left(1+\frac{w}{R}\right)}{\mu_{0} \pi\left(H_{\text {shield }}-h\right)} .
$$

Note that the reluctance $\Re_{3}$ is counted twice in (3) because it appears in the top and in the bottom part of the field pattern.

\section{COMPARISON OF ANALYTICAl Formula AND NUMERICAL RESULTS}

We expect our formula to give good results as far as the assumption of homogeneous magnetic field still holds. Typical $h$ and $w$ dimensions are between 10 and $20 \mathrm{~mm}$ and typical $R_{\text {shield }}$ and $H_{\text {shield }}$ dimensions are not more than five times larger. To obtain the best value for $a$, we have compared expression (3) with numerical methods for the different geometries shown in Table I. Since the value obtained with the analytical approximation for $0.7<a<0.9$ is always within $11 \%$ of the value obtained with numerical methods, $a=0.8$ is used in the rest of the paper. The numerical methods have earlier proved to be exact within 3\% [7]. The numerical calculations involved in this table took some hours in a Pentium III PC at $866 \mathrm{MHz}$ with 256 Mb RAM.

For a fixed $R_{\text {shield }}$, there is an optimum value for $R$ for which $L_{\mathrm{CCC} \text {,eff }}$ is maximum. Fig. 5 shows a good agreement between the results obtained with expression (3) and with

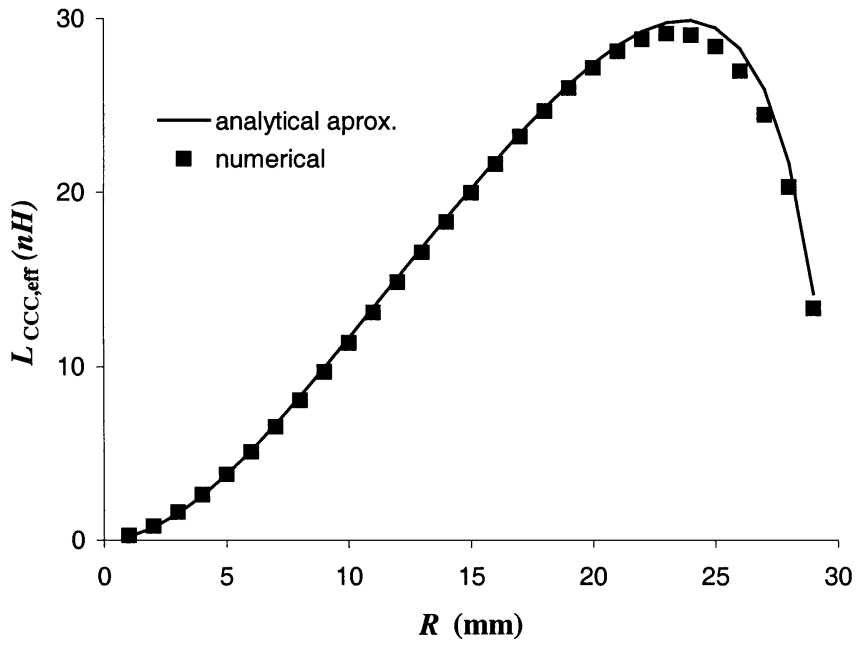

Fig. 5. Value of $L_{\mathrm{CCC} \text {,eff }}$ as a function of $R$ for $h=w=20 \mathrm{~mm}$, $H_{\text {shield }}=80 \mathrm{~mm}$ and $R_{\text {shield }}=50 \mathrm{~mm}$. Squares correspond to numerical calculation based on finite element method. The line corresponds to the analytical approximation deduced in this work with $a=0.8$. The analytical line deviates at most $10 \%$ from the squares.

numerical methods for $h=w=20 \mathrm{~mm}, H_{\text {shield }}=80 \mathrm{~mm}$, and $R_{\text {shield }}=60 \mathrm{~mm}$. In fact, the maximum occurs approximately when the internal area of the CCC, $A_{\text {int }}=\pi R^{2}$, is equal to the area $A_{\text {ext }}$ between the CCC outer side and the shield: $A_{\text {ext }}=\pi R_{\text {shield }}^{2}-\pi(R+w)^{2}$. This criterion can be used as a simple "rule of thumb" to obtain the maximum value of $L_{\mathrm{CCC} \text {,eff }}$. It easily follows from the analytical formula (3) when we make the approximations $\Re_{1} \approx\left(h / \mu_{0} A_{\text {int }}\right)$, $\Re_{2} \approx\left(h / \mu_{0} A_{\text {ext }}\right)$ and $A_{\text {int }}+A_{\text {ext }}=$ constant. The rule of thumb is saying that the minimum reluctance (and hence 


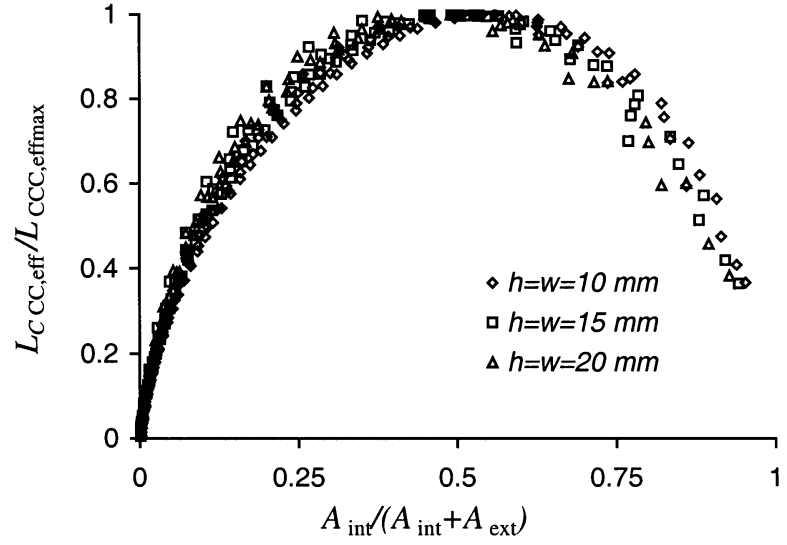

Fig. 6. Numerical calculation of $L_{\mathrm{CCC} \text {,eff }}$ for typical CCC dimensions, normalized to its maximum $L_{\mathrm{CCC} \text {,effmax }}$. In all studied cases, the maximum is reached when $A_{\text {int }}=A_{\text {ext }}$.

the maximum inductance) occurs when $\Re_{1}$ and $\Re_{2}$ have the same contribution to the total reluctance. Fig. 6 shows the value of $L_{\mathrm{CCC} \text {,eff }} / L_{\mathrm{CCC} \text {,effmax }}$ versus $A_{\text {int }} /\left(A_{\text {int }}+A_{\text {ext }}\right)$ as obtained with numerical calculations for different parameters

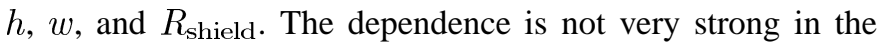
region of the maximum, in all the cases studied, the value of $L_{\mathrm{CCC} \text {,eff }} / L_{\mathrm{CCC} \text {,effmax }}$ was already higher than 0.95 when $A_{\text {int }} /\left(A_{\text {int }}+A_{\text {ext }}\right)$ is between 0.45 and 0.55 .

\section{CONCLUSION}

We have used the theory of magnetic circuits to deduce an approximate formula for the calculation of the effective self-inductance of the superconducting overlapped tube of a CCC inside a superconducting shield. We have validated the accuracy of the formula using numerical methods for typical dimensions of the CCC. By using this analytical formula, there is no need to use numerical methods anymore; hence the design of an optimum $\mathrm{CCC}$ is simplified. Even more, we have derived a general "rule of thumb," namely that the maximum value of the overlapping tube inductance of a CCC inside a superconducting shield occurs when the internal and external areas of the CCC are equal.

\section{ACKNOWLEDGMENT}

The authors would like to thank Prof. D. Gonzalez for his inspiration.

\section{REFERENCES}

[1] I. K. Harvey, "A precise low temperature dc ratio transformer," Rev. Sci. Instrum., vol. 43, pp. 1626-1629, 1972.

[2] B. Jeckelmann and B. Jeanneret, "The quantum Hall effect as an electrical resistance standard," Rep. Prog. Phys., vol. 64, pp. 1603-1655, 2001.

[3] A. Hartland, "Development of a cryogenic current comparator for the measurement of small currents," in Conf. Electromech. Meas. BEMC, vol. 18, 1993, pp. 1-4.

[4] Y. deWilde, F. Gay, F. Piquemal, and G. Geneves, "Measurements of single electron transistor devices combined with a CCC: Progress report," IEEE Trans. Instrum. Meas., vol. 50, pp. 231-234, Apr. 2001.

[5] E. Bartolomé, J. Sesé, G. Rietveld, J. Flokstra, C. Rillo, A. Camón, and H. Rogalla, "1:30 000 cryogenic current comparator with optimum SQUID readout," in CPEM Conf. Dig., 2002, pp. 280-281.

[6] J. Sesé, A. Camón, G. Rietveld, and C. Rillo, "Ultimate current resolution of a cryogenic current comparator," IEEE Trans. Instrum. Meas., vol. 48, pp. 1306-1313, Dec. 1999.
[7] J. Sesé, F. Lera, A. Camón, and C. Rillo, "Calculation of effective inductances of superconducting devices. Application to the cryogenic current comparator," IEEE Trans. Appl. Superconduct., vol. 9, pp. 58-62, Mar. 1999.

[8] G. T. Symm, "Design of a cryogenic current comparator," in Proc. Boundary Elements XIV: Field Problems Applicat., vol. 1, 1992, pp. 519-526.

[9] M. D. Early and K. Jones, "Optimum sensitivity of an externally shielded cryogenic current comparator," IEEE Trans. Instrum. Meas., vol. 46, pp. 459-462, Apr. 1997.

[10] E. T. Frantsuz, "On forward estimate of the sensitivity of a cryogenic current comparator," Metrologia, vol. A85, pp. 847-851, 1998.

[11] S. Marshall and G. G. Skitek, Electromagnetic Concepts and Applications, 3rd ed. Englewood Cliffs, NJ: Prentice-Hall, 1990, pp. 276-283.

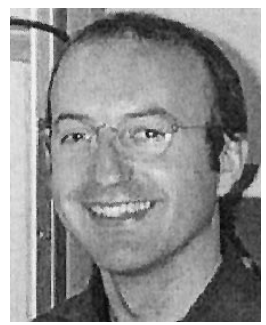

Javier Sesé is from Binéfar, Spain. He received the M.Sc. and Ph.D. degrees in physics from Zaragoza University, Zaragoza, Spain, in 1993 and 1999, respectively.

Since 1993, he has been with the Institute of Material Science of Aragon (ICMA), CSIC-University of Zaragoza. He has been working on electrical quantum metrology, applied superconductivity, and low-temperature instrumentation. He is currently in a post-doctorate stay at the University of Twente, Enschede, The Netherlands, where he works on microelectromechanical systems for precision applications.

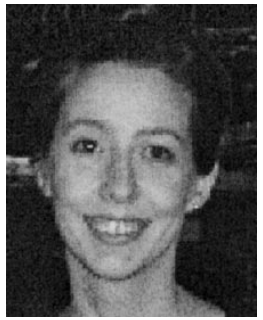

Elena Bartolomé was born in Zaragoza, Spain, on August 21, 1974. She received the M.Sc. degree in physics from the University of Zaragoza, Zaragoza, in 1997, and the Ph.D. degree in applied physics from the University of Twente, Enschede, The Netherlands, and the University of Zaragoza in 2002.

She has worked on low Tc superconducting sensors (SQUIDs, cryogenic current comparator systems), with application in quantum metrology. At present, she has a post-doctorate position at the Institute of Material Sciences Barcelona (ICMAB), Barcelona, Spain, where she works on YBCO power applications and the development of chemically prepared coated conductors.

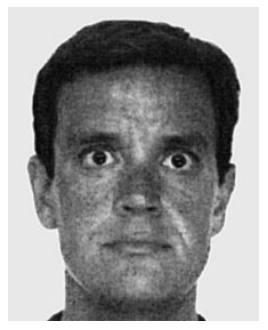

Agustín Camón was born in Peñaranda de Bracamonte, Spain, on March 17, 1962. He received the Ph.D. degree in physics from the University of Zaragoza, Spain, in 1996.

Since 1990, he has been with the Technical Staff at the Institute of Material Science of Aragon (ICMA), CSIC-University of Zaragoza, where he has been working on quantum metrology, applied superconductivity, and low temperature instrumentation.

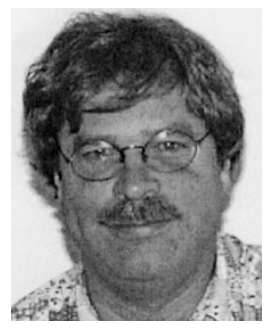

Jaap Flokstra received the M.Sc. degree in applied physics from the University of Delft, Delft, The Netherlands, and the Ph.D. degree in applied physics from the University of Twente, Twente, The Netherlands, in 1971 and 1977, respectively.

He has been an Associate Professor in the Department of Applied Physics, University of Twente, since 1985. His current interest is in the development and application of low and high Tc SQUID-based sensors in areas as biomagnetism, particle detection, metrology, and very fast electronics. 


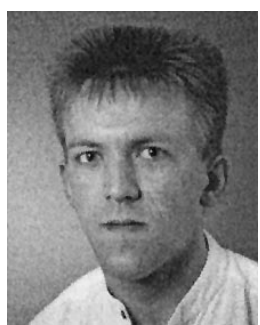

Gert Rietveld was born in The Netherlands in 1965. He received the M.Sc. and Ph.D. degres in physics from the Delft University of Technology, Delft, The Netherlands, in 1988 and 1993, respectively.

In 1993, he joined the NMi Van Swinden Laboratorium, Delft, where he works in the DC/LF group of the Electrical Metrology Department. He is involved in the development of electrical quantum standards, especially the quantum Hall resistance standard and the current standard based on single electron tunnelling effects. He is also active in evaluation of "self-calibrating" instruments.

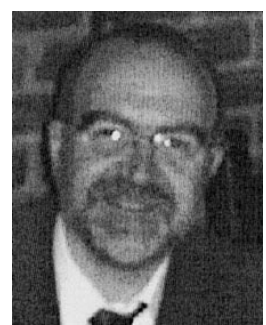

Conrado Rillo was born in Alcañiz, Spain, on May 8,1957 . He received the M.Sc. degree in physics and the Ph.D. degree from the University of Zaragoza, Zaragoza, Spain, in 1982 and 1986, respectively.

Since 1987, he has been with the research staff at the Institute of Material Science of Aragon (ICMA), CSIC-University of Zaragoza, where he has been working in the fields of materials research and advanced instrumentation. He is presently Research Professor and Head of the Low Temperature Physics Department at ICMA. His current research interests include magnetic and superconducting materials, quantum metrology and applied superconductivity. He is also responsible for the Scientific Instrumentation Service of the University of Zaragoza. 